\title{
VIRTUAL SCREENING OF BETA-SECRETASE 1 (BACE1) INHIBITORS IN THE INDONESIAN HERBAL DATABASE AS USING AUTODOCK AND AUTODOCK VINA
}

\author{
ASTI ANNA TANISA, REZI RIADHI, ARRY YANUAR* \\ Department of , Faculty of Pharmacy, Universitas Indonesia, Depok, Indonesia. Email: arry.yanuar@ui.ac.id
}

Received: 20 April 2017, Revised and Accepted: 13 July 2017

ABSTRACT

Objective: Alzheimer's is a neurodegenerative disease caused by the accumulation of senile plaque in the brain that affects neuronal system leading to a less sensitive cellular response from neurons. Previous research has found that beta-secretase 1 (BACE1) plays an important role in the senile plaque formation, become a target in Alzheimer's medication.

Methods: In this study, virtual screening of BACE1 inhibitors on the Indonesian Herbal Database was done using AutoDock and AutoDock Vina. The screening was validated using the directory of useful decoys: Enhanced database. Parameters for validation process of AutoDock and AutoDock Vina are enrichment factor (EF), receiver operating characteristics, and area under the curve (AUC).

Results: The dimensions of grid boxes were $30 \times 30 \times 30$ (AutoDock) and $11.25 \times 11.25 \times 11.25$ (AutoDock Vina). The EF $1 \%$ and AUC values obtained from the AutoDock are 7.74 and 0.73 , respectively, and in the AutoDock Vina are 4.6 and 0.77 , respectively. Based on the virtual screening results, the top six compounds obtained using AutoDock (binding energy ranging from $-7.84 \mathrm{kcal} / \mathrm{mol}$ to $-8.79 \mathrm{kcal} / \mathrm{mol}$ ) include: Azadiradione, cylindrin, lanosterol, sapogenin, simiarenol, and taraxerol. The top seven compounds (binding energy ranging from $-8.8 \mathrm{kcal} / \mathrm{mol}$ to $-9.4 \mathrm{kcal} / \mathrm{mol}$ ) obtained using AutoDeck Vina include: Bryophyllin A, diosgenin, azadiradione, sojagol, beta-amyrin, epifriedelinol, and jasmolactone C.

Conclusions: Only azadiradione was obtained from the virtual screening conducted using both types of software; it interacts with the active region in BACE1 at residue Trp 76 (AutoDock result) and Thr 232 (AutoDock Vina result).

Keywords: Virtual screening, Herbal database, BACE1, Molecular docking

(C) 2017The Authors. Published by Innovare Academic Sciences Pvt Ltd. Thisisan open access article under the CC BYlicense (http://creativecommons. org/licenses/by/4. 0/) DOI: http://dx.doi.org/10.22159/ajpcr.2017.v10s5.23119

\section{INTRODUCTION}

Alzheimer's disease is the most common type of dementia (60-70\% of all cases), and it can cause the death of the neurons in the brain that regulate memory and knowledge [1]. In 2010, 35.6 million people, worldwide, suffered from dementia, and it is expected that there will be 7.7 million new cases each year [1]. The progression of dementia is very rapid, so fast action is required to prevent Alzheimer's disease and to decrease the prevalence of dementia [1]. Current treatment for Alzheimer's is still unable to prevent the disease from progressing; thus, the discovery of new drugs to stop the neurodegenerative process is urgently needed [2]. The enzyme beta-secretase 1 (BACE1, memapsin-2, Asp-2; beta-site APP-cleaving enzyme 1) is important in the formation of senile plaque; treatment approach can be done by targeting its active sites (Asp 32 and Asp 228) to block the activity of enzyme [3]. This treatment approach cannot be used with other types of secretases, such as gamma-secretase, because gamma-secretase inhibition can have some side effects that affect the activity of the Notch receptor and beta-secretase 2 (BACE2, memapsin-1, Asp-1; beta-site APP-cleaving enzyme 2), which is a homolog of BACE1 that is expressed in small numbers in neurons, the effect of BACE2 on the development of Alzheimer's disease remains unclear [2].

A 2012 study about the structure-based design of iminohydantoin as a BACE1 inhibitor using a computational method is one of the computer-based studies that have indicated that BACE1 can reduce the pathogenesis of Alzheimer's disease [4]. Over the past 15 years, the source of drug discovery has been high-throughput screening on compound libraries; however, this method only yields a small number of drugs that actually work [5]. Nowadays, computer-based drug design is preferred over the conventional method because it is fast and it does not require a lot of time; it is also cost-effective, and it can reduce the number of possible compounds for in vitro evaluation [6].

Herbal medicines contain active compounds that can be effective as medications. Indonesia is a tropical country with a wide variety of herbal medicines [7], so the virtual screening in the present study was performed using the Herbal Database (HerbalDB) compiled by the Faculty of Pharmacy at the University of Indonesia. The database contains three-dimensional (3D) structures of 1481 compounds from Indonesia's herbal medicines that have the possibility of being BACE1 inhibitors [8]. The present study used AutoDock and AutoDock Vina as the main software programs for virtual screening and molecular docking. Molecular docking is a computational method that can predict the position and correct conformation between a ligand and a receptor when the two molecules form a stable complex, thus providing knowledge on the binding affinity between them [9]. Software for molecular docking utilizes the principle of correct conformation to search for receptor-ligand bonds using two types of algorithms: Search algorithms

andscoringfunctions [9].Searchalgorithmsprovidea variety of possible positions for receptor-ligand bonds and scoring functions rank different positions and locations of the ligands obtained from the search algorithm [9]. In the scoring function, the score represents the thermodynamic interaction between the ligand-receptor bonds to distinguish them from other bonds [9]. Virtual screening is another method that allows researchers to identify possible lead compounds for drug development or to select some compounds in silico [10]. Validation for AutoDock and AutoDock Vina was performed by calculating the root mean square deviation (RMSD), the enrichment factor (EF), and the receiver operating characteristics (ROC) [8]. The results obtained from this approach are compounds that have the possibility of being BACE1 inhibitors. 


\section{METHODS}

\section{Tools and materials}

This study used the following software programs: Open Babel (The Blueobelisk Group, San Diego, California), PyMOL (Delano Scientific LLC, San Carlos, California), AutoDock Tools (The Scripps Research Institute, San Diego, California), AutoDock 4.0 (The Scripps Research Institute, San Diego, California), AutoDock Vina, LigandScout, LigPlot+, UCSF Chimera, and PyRx.

The following materials were used in this study: The 3D structure of macromolecule BACE1, which was downloaded from the RCSB Protein Data Bank (PDB), the 3D structures of the positive control and the negative control, which were downloaded from directory of useful decoys-enhanced (DUD-E), and the 3D structures of Indonesian plant compounds, which were downloaded from HerbalDB.

\section{METHODS}

The ligand that has the potential to be a drug was downloaded from HerbalDB. The positive control was obtained by separating the 3D structures of the macromolecules 4B1D from the PDB, which were downloaded from DUD-E, and the negative control, which was also downloaded from DUD-E.

The positive and negative controls used in the validation process were downloaded from DUD-E in the form of *.mol2. Then, the file was inputted into Open Babel software and was validated using PyRx. It was then minimized using force field MMFF94 and changed to the *.pdbqt format. At that point, the file was validated and added to the PyRx workspace.

Macromolecule 4B1D from the PDB is a protein that contains a solvent molecule and non-standard residue, so it has to be separated using AutoDock Tools. In the next step, the macromolecule structure was optimized, which involved removing a water molecule, adding a hydrogen atom, repairing the change by adding a partial load of Gasteiger charge, repairing the AutoDock force field, and applying minimization. After that the virtual screening method was validated using the positive and negative controls to the target macromolecule. The validation result was then optimized by obtaining the EF and ROC values. The docking parameter varies to generate a lower free energy score, a more homogenous cluster distribution, and a smaller RMSD score $(<2 \AA)$. Next, virtual screening was performed using PyRx, which was then followed by the docking stage, which involved searching the 3D structures from HerbalDB in the form of *.mol2 and converting the two dimensional (2D) structures of the drug candidate compounds using open Babel software. The docking result parameter was measured using the free energy ties, the inhibition constant for inhibitor, and the existing interactions. In the past step, the visualization was conducted using PyMOL, LigandScout, and LigPlot+.

\section{RESULTS AND DISCUSSION}

Macromolecule ID was chosen if it bonded with a ligand; the bond between the ligand and the macromolecule results in the macromolecule conformation used in the molecule docking stage to become an active conformation so it will be easier for the macromolecule to interact with the ligand [11]. Another criterion used to choose a target protein is that the protein needs to have a complete amino acid sequence, and the resolution should be $<2 \AA$ [11]. The present research study uses a target protein with a 4B1D code, which is polypeptide chain A from the BACE1 enzyme.

\section{Optimization of the protein structure and determination of the} binding site

Optimization was done by separating the macromolecule from the solvent and non-standard residue, adding a polar hydrogen atom, and repairing the force field by adding a Gasteiger charge. Then, the center of bond was searched for using center on ligand in the AutoDock tools.
Thus, the coordinates of the center of the bond are: $x=5.673 ; y=1.606$; and $\mathrm{z}=-21.428$.

In the next step, optimization of the protein structure was performed using a redocking cocrystal, which was previously separated from the macromolecule. Then, the RMSD of the cocrystal caused by redocking toward the newly separated cocrystal was measured. The grid boxes used in this study had the following dimensions: $30 \times 30 \times 30$ (AutoDock) and $11.25 \times 11.25 \times 11.25$ (AutoDock Vina).

The redocking process was used to determine the accuracy of the structure from the prediction results [12] and the accuracy of the RMSD parameters, which would verify the software's ability to simulate the structure so that the simulation results would not be very different from the initial structure; this confirms that the structure is stable [13]. A higher RMSD score might indicate inaccuracy of a prediction for a large molecule, which is precisely placed according to the initial cocrystal position; however, this does not apply to a small molecule that can easily generate a small RMSD score even when the molecule is randomly placed [12]. The RMSD parameter cannot be the standard used to determine the best grid box because it depends on the weight of the molecule, and this method is considered biased because it does not take into account the changes in the protein conformations in the ligand bonds [12].

\section{Validation of the virtual screening method using AutoDock}

The AutoDock validation results validated the bond energy between the positive control and the negative control. The result was saved in a *.csv file format; it consisted of the bond energy value, the intermolecular energy, the internal energy, the torsion energy, and the free energy. The validation parameters included EF, ROC, and area under the curve (AUC). EF measures the number of active compounds that are found in the compound hit list by considering the fraction of inactive compounds (negative control) [12]. EF is valid if the score is close to or above the random score (EF score $>1$ ) [14]. In the present research study, values were calculated for EF $1 \%, 5 \%, 10 \%$, and $20 \%$. According to the results of the positive and negative control compound screening, the $\mathrm{EF}$ score resulting from the $30 \times 30 \times 30$ grid box is the largest of all the EF scores (EF 1\% value of 7.74 for AutoDock and 4.6 for AutoDock Vina).

The EF calculation has its own limitations; it is highly dependent on active molecules from the screened database. Thus, a second parameter, ROC, must be used to determine the accuracy of the virtual screening method [12]. As seen in Fig. 1, all the grid boxes produced curves above the random line. However, the $30 \times 30 \times 30$ grid box met all the ROC score requirements, and it produced the most ideal ROC curve.

Next, the AUC, which provides more specific details about the validation of the virtual screening method, was measured [12]. The smallest grid, which is $30 \times 30 \times 30$, generated the most ideal score based on the AUC calculation.

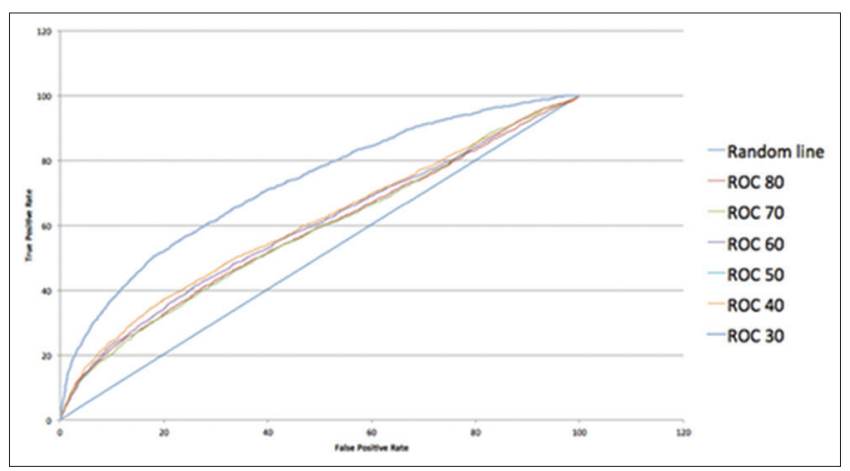

Fig. 1: Receiver operating characteristics curve validation for AutoDock 
Validation of the virtual screening method using AutoDock Vina The AutoDock Vina validation results show the bond energy between the positive control and negative controls [8]. The results were saved in a *.csv file format; this included the affinity bond value, the RMSD upper bound (RMSD/ub), and the RMSD lower bound (RMSD/lb). The following validation parameters were used: EF, ROC, and AUC [12]. The values for $\mathrm{EF} 1 \%, 5 \%, 10 \%$, and $20 \%$ were calculated. The score of $\mathrm{EF}$ $1 \%$ resulted by a $30 \times 30 \times 30$ grid box, and it is the largest of all the EF scores.

As seen in Fig. 2, all the grid boxes produced curves above the random line, but the $30 \times 30 \times 30$ grid box produced the most ideal ROC curve.

The ROC score of the sixth grid box is higher than ideal score ( $>0.5)$; the $30 \times 30 \times 30$ grid box is the largest grid, but the $26.25 \times 26.25 \times 26.25$ grid had the most ideal ROC score.

\section{Virtual screening using AutoDock}

The file output produced by AutoDock in the.dlg format can be viewed using AutoDock tools [8]. The energy docking and constant inhibition results from the*.dlg about file showing the top six potential compounds are presented in Table 1.

\section{Virtual screening using AutoDock Vina}

The top seven compounds obtained from the virtual screening using AutoDock Vina (contained in the ligand-out.pdbqt file) are presented in Table 2. The docking results using AutoDock Vina did not generate

Table 1: Top six compounds from virtual screening using AutoDock

\begin{tabular}{lll}
\hline Compounds & Energy (kcal/mol) & $\begin{array}{l}\text { Inhibition constant } \\
\text { at 298.15oK }\end{array}$ \\
\hline Azadiradione & -8.79 & $358.64 \mathrm{nM}$ \\
Cylindrin & -8.74 & $390.38 \mathrm{nM}$ \\
Lanosterol & -8.69 & $429.00 \mathrm{nM}$ \\
Taraxerol & -8.53 & $561.79 \mathrm{nM}$ \\
Simiarenol & -8.40 & $696.97 \mathrm{nM}$ \\
Sapogenin & -7.84 & $1.79 \mathrm{uM}$ \\
\hline
\end{tabular}

Table 2: Top seven compounds from the virtual screening using AutoDock Vina

\begin{tabular}{ll}
\hline Compounds & Energy (kcal/mol) \\
\hline Bryophyllin A & -9.4 \\
Diosgenin & -9.1 \\
Azadiradione & -9.0 \\
Sojagol & -8.9 \\
Beta amyrin & -8.8 \\
Epifriedelinol & -8.8 \\
Jasmolactone C & -8.8 \\
\hline
\end{tabular}

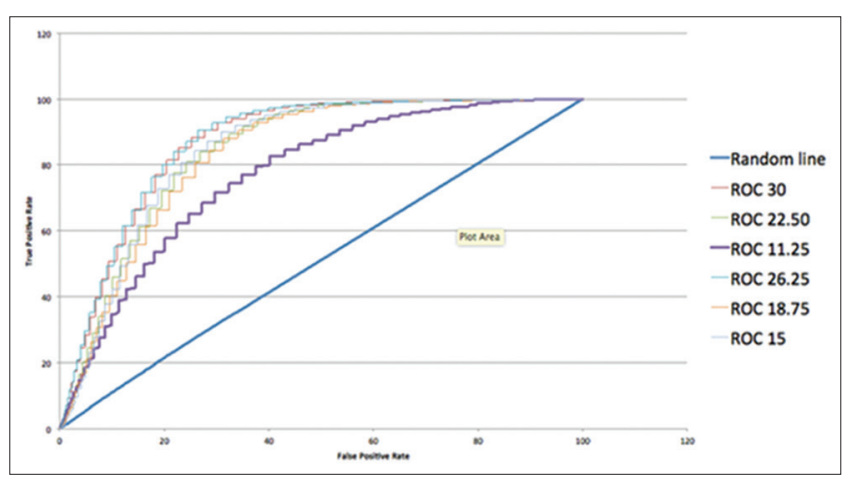

Fig. 2: Receiver operating characteristics curve validation using AutoDock Vina data on the inhibition constant due to the *.pdbqt format of the data output.

The general structure of a BACE1 inhibitor is presented in Fig. 3 [15]. The substituents in R1 and R2 determine the activity of a biological molecule and how it would potentially work as a BACE1 inhibitor [15]. One study reported that the structures of natural BACE1 inhibitors, such as biphenyl, pentacyclene, benzopiran/benzofuran, and stilbene, and stilben-look-alikes, are similar; they all contain an aromatic ring [16]. Based on visualization of 2D structures, these five compounds have a nearly similar pentacyclic structure. They are also included in the class of triterpenoids.

Visualization of the virtual screening results

Only one compound, azadiradione, was identified in the virtual screening results for both AutoDock and AutoDock Vina. That compound is included in the class of triterpenoids (limonoids), and it originates in the plant Azadirachta indica, which is a member of the Meliaceae family. The superposition of the structure of azadiradione from AutoDock and AutoDock Vina (gold) with cocrystal (blue) shows a structural similarity, so it is not enough to use as a reference that azadiradione has the potential to be a BACE1 inhibitor. Fig. 4 shows the visualization of the azadiradione superposition using Chimera. In the 2D structure, visualization result of 12 filtered compounds, seven of the 12 compounds (e.g., diosgenin, lanosterol, cylindrin, epidriedelinol, teraxerol, simiarenol, and beta-amyrin) contain a cyclic carbon group

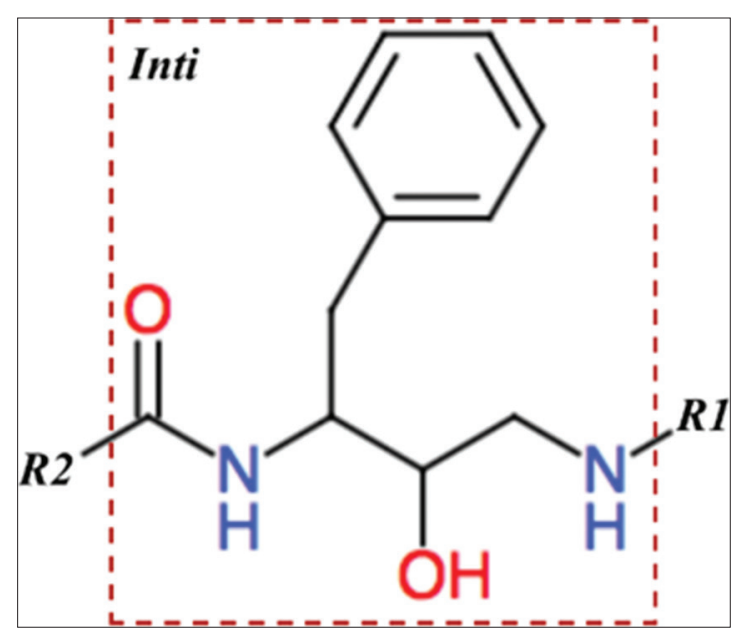

Fig. 3: Structure of a BACE1 inhibitor [15]

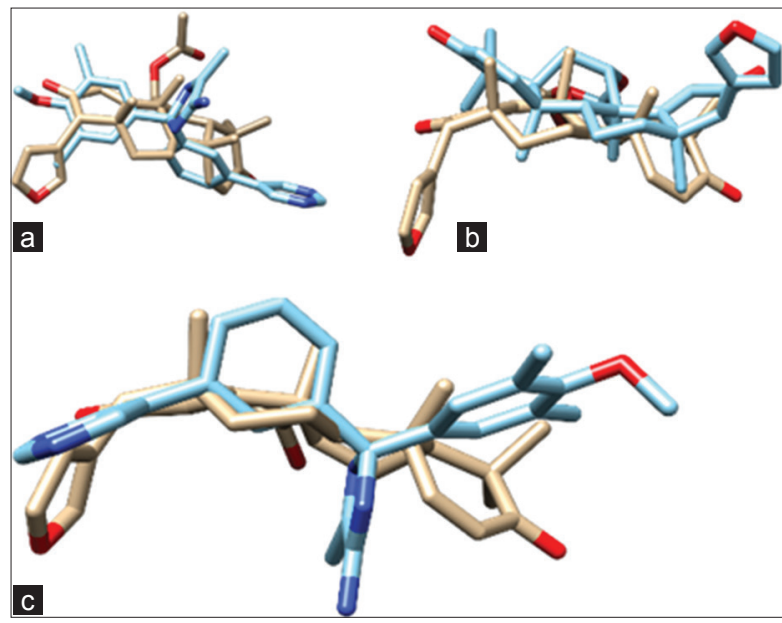

Fig. 4: (a-c) Superposition of the structure of azadiradione from AutoDock and AutoDock Vina (gold) with cocrystal (blue) 
with one group of $-\mathrm{O}$ or $-\mathrm{OH}$; hence, this might increase the lipophilicity value of these compounds.

\section{Visualization of the virtual screening results}

Only one compound, azadiradione, was identified in the virtual screening results for both AutoDock and AutoDock Vina. That compound is included in the class of triterpenoids (limonoids), and it originates in the plant $A$. indica, which is a member of the Meliaceae family. The superposition of the structure of azadiradione from AutoDock and AutoDock Vina (gold) with cocrystal (blue) shows a structural similarity, so it is not enough to use as a reference that azadiradione has the potential to be a BACE1 inhibitor. Fig. 4 shows the visualization of the azadiradione superposition using Chimera. In the 2D structure, visualization result of 12 filtered compounds, seven of the 12 compounds (e.g., diosgenin, lanosterol, cylindrin, epidriedelinol, teraxerol, simiarenol, and beta-amyrin) contain a cyclic carbon group with one group of $-\mathrm{O}$ or $-\mathrm{OH}$; hence, this might increase the lipophilicity value of these compounds.

\section{Ligand interaction with residue BACE1}

The results of the ligand interaction with residue BACE1, obtained from the virtual screening using either AutoDock or AutoDock Vina, are presented in Tables 3 and 4, respectively. As seen, there is a difference between the virtual screening results for azadiradione using AutoDock and AutoDock Vina. The AutoDock screening result shows that the hydrogen bond in residue Trp 76 occurred because atom 01 from azadiradione interacted with atom $\mathrm{N}$ within the structure of Trp 76 and caused atom 0 in azadiradione to act as a hydrogen acceptor. In addition, this reaction occurred within the range of $3.21 \AA$. A hydrophobic interaction also occurred between the azadiradione complex and BACE1. A hydrophobic interaction refers to self-bonding among non-polar compounds in a specific environment. A different result was obtained for azadiradione docking using AutoDock Vina. The azadiradione interaction with atom 0 produced a hydrogen bond in residue Thr 232, which caused atom 0 to act as a hydrogen acceptor in the range of $2.82 \AA$. Moreover, a hydrophobic bond also formed among the non-polar compounds within the BACE1 bonding site. In the AutoDock Vina screening, the azadiradione that resulted from docking produced a higher energy bond (kcal $/ \mathrm{mol}$ ) than one produced with AutoDock, which was $-9.0 \mathrm{kcal} / \mathrm{mol}$ in AutoDock Vina and $-8.79 \mathrm{kcal} / \mathrm{mol}$ in AutoDock.

This finding is consistent with the results reported in a previous study, which stated that AutoDock Vina can significantly increase the average accuracy of bonding prediction in comparison to AutoDock [8]. The energy bond results $(-7.85 \mathrm{kcal} / \mathrm{mol})$ for azadiradione are better than cocrystal energy bond (6TG1505) results for this compound in redocking using AutoDock. This result is very similar to the energy bond result $(-9.20 \mathrm{kcal} / \mathrm{mol})$ obtained with AutoDock. Jasmolactone C is the only compound that shows a similar hydrogen bond with a ligand interaction result of 6TG1505 in residue Asp 228, which is an active site of BACE1. The bonding within hydrogen atoms occurs because atom 0 from Asp 228 interacts with atom 0 on the jasmolactone $C$ structure, and this bond is formed at a distance of $3.18 \AA$.

Moreover, the AutoDock Vina docking results for jasmolactone $\mathrm{C}$ show that this compound also produces an energy bond ( $\mathrm{kcal} / \mathrm{mol})$ that is not very different from the cocrystal redocking results, which are $-8.80 \mathrm{kcal} / \mathrm{mol}$ for jasmolactone $\mathrm{C}$ and $-9.20 \mathrm{kcal} / \mathrm{mol}$ for azadiradione. The azadiradione that resulted from docking using AutoDock Vina produces a bonding site that is similar to the bonding site seen in jasmolactone C (from docking using AutoDock Vina), which is on residue Thr 232 from the active site of BACE1.

\section{CONCLUSION}

The optimum parameters from the virtual screening validation using AutoDock include: The $30 \times 30 \times 30$ grid box with an AUC of 0.73 and an EF $1 \%$ value of 7.74 ; for AutoDock Vina the optimum parameters include: The $30 \times 30 \times 30$ grid box with an AUC of 0.87 and an EF $1 \%$ value of 4.6. Six compounds were obtained from the AutoDock virtual screening: Azadiradione, cylindrin, lanosterol, taraxerol, simiarenol, and sapogenin. Seven compounds were obtained from the AutoDock Vina virtual screening: Bryophyllin A, diosgenin, azadiradione, sojagol, beta-amyrin, epifriedelinol, and jasmolactone C. Both AutoDock and AutoDock Vina yielded azadiradione as a possible hit compound for a BACE1 inhibitor. When docking with AutoDock, azadiradione binds

Table 3: Amino acid residue interactions from AutoDock docking

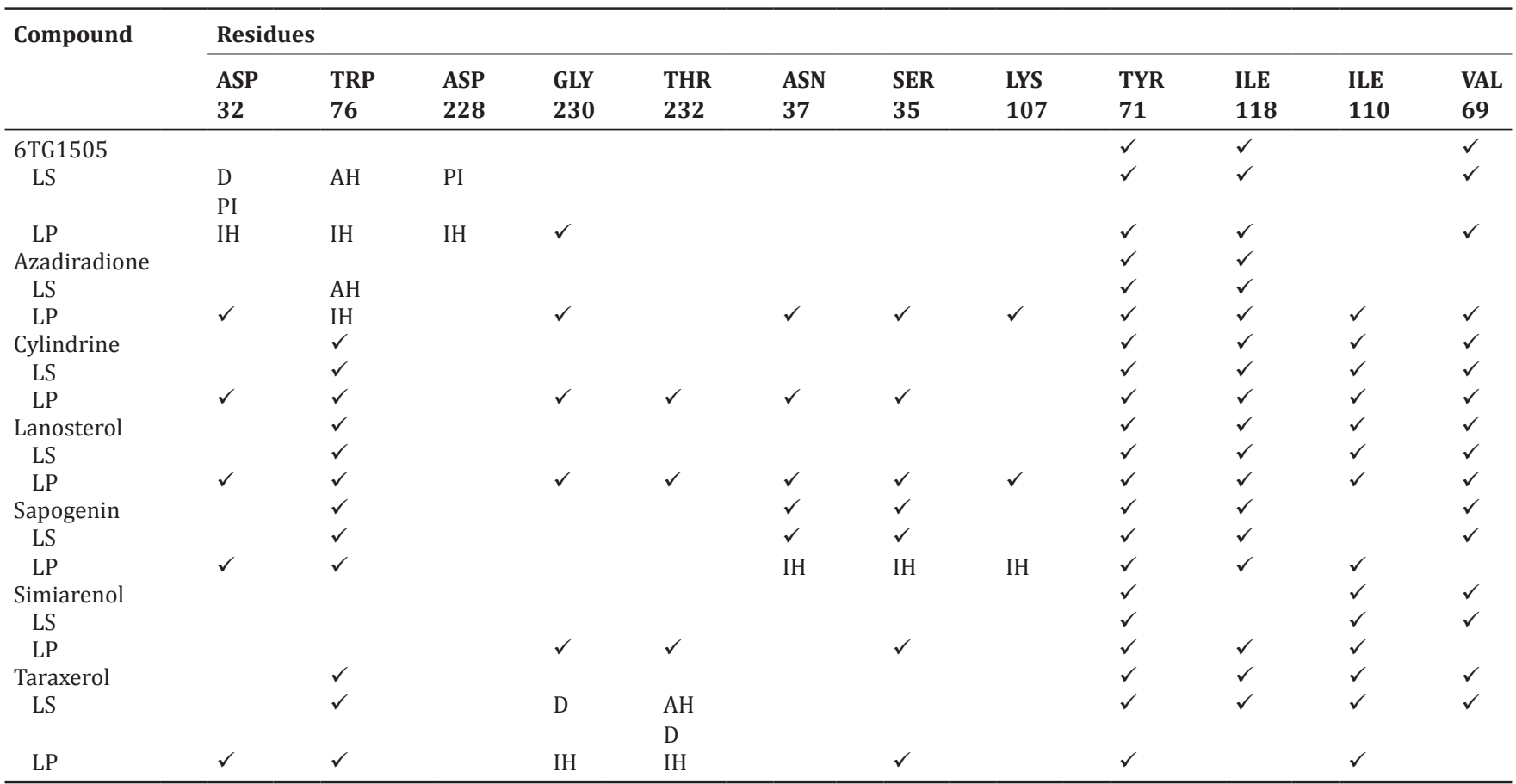

LS: LigandScout, LP: LigPlot+, v: Hydrophobic interaction, D: H Donor, AH: H acceptor, PI: Positive ionizable, IH: Hydrogen binding 
Table 4: Amino acid residue interactions from AutoDock Vina docking

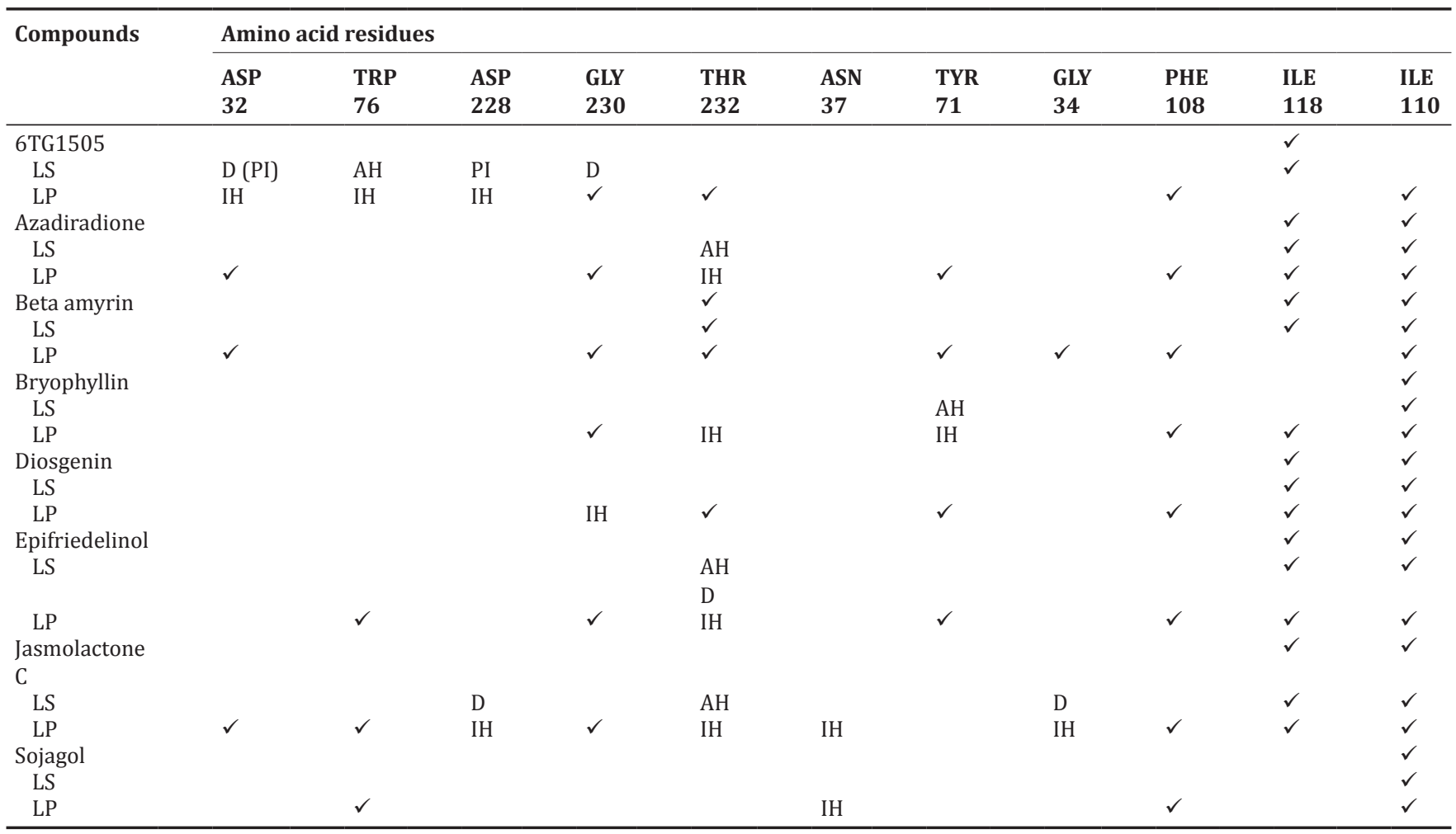

LS: LigandScout, LP: LigPlot+, v: Hydrophobic interaction, D: H Donor, AH: H acceptor, PI: Positive ionizable, IH: Hydrogen binding

with Trp 76; when docking with AutoDock Vina, azadiradione binds with Thr 232.

\section{ACKNOWLEDGMENTS}

The authors would like to acknowledge the financial support of PITTA from DRPM UI.

\section{REFERENCES}

1. Vyas V, Jain A, Jain A, Gupta A. Virtual screening: A fast tool for drug design. Sci Pharm 2008;76(3):333-60.

2. Gravenfors Y, Viklund J, Blid J, Ginman T, Karlström S, Kihlström J. New aminoimidazoles as $\beta$ - Secretase (BACE-1) inhibitors showing Amyloid- $\beta$ (A $\beta$ ) lowering in brain. J Med Chem 2012;55(21):9297-311.

3. Hwang EM, Ryu YB, Kim HY, Kim DG, Hong SG, Lee JH, et al. BACE1 inhibitory effects of lavandulyl flavanones from Sophora flavescens. Bioorg Med Chem 2008;16:6669-74.

4. Cumming JN, Smith EM, Wang L, Misiaszek J, Durkin J, Iserloh U. Structure based design of iminohydantoin BACE1 inhibitors: Identification of an orally available, centrally active BACE1 inhibitor. Bioorg Med Chem Lett 2012;22(7):2444-9.

5. Alvarez J, Shoichet B. Virtual Screening in Drug Discovery. Boca Raton: CRC Press Taylor \& Francis Group; 2005.

6. Yanuar A, Mun'im A, Lagho AB, Syahdi RR, Rahmat M, Suhartanto H. Medicinal plants database and three dimensional structure of the chemical compounds from medicinal plants in Indonesia. Int J Comput Sci 2011;8(5):180-3

7. Pribadi ER. Pasokan dan permintaan tanaman obat Indonesia serta arah penelitian dan pengembangannya. Perspectives 2009;8(1):52-64.
8. Yanuar A. Penambatan Molekular: Praktek Dan Aplikasi Pada Virtual Screening. Depok: Fakultas Farmasi, Universitas Indonesia; 2012. p. $39-60$.

9. Cerqueira NM, Gesto D, Oliveira EF, Santos-Martins D, Brás NF, Sousa SF. Receptor-based virtual screening protocol for drug discovery. Arch Biochem Biophys 2015;582:56-7.

10. Lemke TL, Williams DA, Roche VF, Zito SW. Foye's principles of medicinal chemistry. $6^{\text {th }}$ ed. USA: Lippincott Williams \& Wilkins; 2008.

11. Fisher L, Varma S, Chen D. Creating a Smart Virtual Screening Protocol, Part I : Preparing the Target Protein. Study; 2005. p. $1 . \quad$ Pharmaceutical Case

12. Kirchmair J, Markt Æ, Distinton S, Wolber Æ. Evaluation of the performance of 3D virtual screening protocols: RMSD comparisons, enrichment assessments, and decoy selection - What can we learn from earlier mistakes ? J Comput Aided Mol 2008;22(3-4):213-8.

13. Kaur J, Goyal S, Sharma S, Hamid R, Grover A. Mechanistic insights into mode of action of potent natural antagonists of BACE-1 for checking Alzheimer's plaque pathology. Biochem Biophys Res Commun 2014;443(3):1054-9.

14. Vinh NB, Simpson JS, Scammells PJ, Chalmers DK. Virtual screening using a conformationally flexible target protein: Models for ligand binding to p38 $\alpha$ MAPK. J Comput Aided Mol Des 2012;26(4):409-23.

15. Huang D, Liu Y, Shi B, Li Y, Wang G, Liang G. Comprehensive 3D-QSAR and binding mode of BACE-1 inhibitors using R-group search and molecular docking. J Mol Graph Model 2013;45:65-83.

16. Youn K, Lee J, Yun E, Ho C. Short communications biological evaluation and in silico docking study of $\gamma$-linolenic acid as a potential BACE1 inhibitor. J Funct Foods 2014;10:187-91. 